\title{
Analytical description of nonlinear cosmic ray scattering: isotropic and quasilinear regimes of pitch-angle diffusion
}

\author{
A. Shalchi, T. Škoda, R. C. Tautz, and R. Schlickeiser \\ Institut für Theoretische Physik, Lehrstuhl IV: Weltraum- und Astrophysik, Ruhr-Universität Bochum, 44780 Bochum, Germany \\ e-mail: andreasm4@yahoo.com
}

Received 24 June 2009 / Accepted 21 August 2009

\section{ABSTRACT}

\begin{abstract}
Aims. We investigate pitch-angle scattering, which is a fundamental process in the physics of cosmic rays. Methods. By employing the second-order quasilinear theory, the pitch-angle Fokker-Planck coefficient is calculated analytically for the first time.

Results. We demonstrate that for sufficiently strong turbulence the pitch-angle Fokker-Planck coefficient is isotropic. The derived results can be used to compute the parallel mean free path for all forms of the turbulence spectrum. We also consider applications, namely the transport of solar energetic particles and the propagation of cosmic rays in the Galaxy.

Conclusions. The previously used assumption of isotropic pitch-angle diffusion is indeed correct for sufficiently strong turbulence. An analytical description of nonlinear particle scattering is possible.
\end{abstract}

Key words. acceleration of particles - diffusion - cosmic rays - Magnetohydrodynamics (MHD) - turbulence interplanetary medium

\section{Introduction}

Here, we revisit the problem of charged particle transport in MHD turbulence. Particle transport is described by the diffusion tensor $\kappa_{i j}$ in the case of diffusive propagation. For certain parameters regimes (for a detailed discussion see Shalchi \& Dosch 2009), one expects isotropic scattering. In this case the tensor is given by $\kappa_{i j}=\kappa \delta_{i j}$. In addition to the turbulent magnetic fields $\delta B_{i}$ we expect the existence of a non-vanishing mean magnetic field $\left\langle B_{i}\right\rangle=B_{0} \delta_{i z}$. The latter parameter breaks the symmetry of the physical system leading to different diffusion coefficients along and across the mean magnetic field. For not too strong turbulent fields we expect $\kappa_{\perp} \ll \kappa_{\|}$. In this case the parallel spatial diffusion coefficient $\kappa_{\|} \equiv \kappa_{z z}$ controls the particle motion.

An important example for the application of diffusion theory is the propagation and acceleration of charged cosmic rays (for a review see Shalchi 2009b). The investigation of these processes is relevant for different physical systems. Some examples are the solar corona (see, e.g., Fletcher 1997; Gkioulidou et al. 2007), the heliosphere (e.g., Dröge 2000; Shalchi et al. 2006; Alania \& Wawrzynczak 2008), the interstellar medium (see, e.g., Yan \& Lazarian 2002; Shalchi \& Schlickeiser 2005), and shock waves (see, e.g., Zank et al. 2000; Li et al. 2003; Li et al. 2005; Zank et al. 2006).

The parallel mean free path $\lambda_{\|}$of the charged particle is related to the parallel spatial diffusion coefficient $\kappa_{\|}$via $\lambda_{\|}=3 \kappa_{\|} / v$ and can be expressed as an integral over the inverse pitch-angle Fokker-Planck coefficient $D_{\mu \mu}$ (see, e.g., Earl 1974)

$\lambda_{\|}=\frac{3 v}{8} \int_{-1}^{+1} \mathrm{~d} \mu \frac{\left(1-\mu^{2}\right)^{2}}{D_{\mu \mu}(\mu)}$

with the pitch-angle cosine $\mu=v_{\|} / v$ and the particle velocity $v$.

The first approach to compute the parameter $D_{\mu \mu}$ was the application of perturbation theory also known as quasilinear theory (QLT, Jokipii 1966). In the years after QLT had been developed, it was noticed that the theory is not able to describe pitch-angle scattering at $90^{\circ}$ (corresponding to $\mu=0$ ) correctly. This problem, which is known as the $90^{\circ}$-scattering problem, was then investigated in numerous papers (see, e.g., Jones et al. 1973; Völk 1973; Owens 1974; Völk 1975; Goldstein 1976; Jones et al. 1978). In these articles, QLT has been improved by replacing unperturbed orbits by more appropriate models. However, some of these previous theories do not provide agreement with simulations or they are difficult to apply due to mathematical problems (see Shalchi 2009a,b). More recently, a second order quasilinear theory (SOQLT, Shalchi 2005a) was developed. This theory is in good agreement with test-particle simulations (for a detailed comparision between SOQLT and simulations we refer to Shalchi 2007) and is mathematically tractable. Furthermore, Tautz et al. (2008) have demonstrated that SOQLT can reproduce the simulations of Giacalone \& Jokipii (1999) performed for isotropic turbulence.

In previous applications of nonlinear theories for pitch-angle scattering and parallel spatial diffusion, only numerical results have been derived due to mathematical intractability. Analytical forms of these parameters are, however, very useful for different astrophysical applications. It is the purpose of the present article to investigate the SOQLT analytically for the first time. In previous articles, simple models have been used without justification. In the theory of diffusive shock acceleration, for instance, it was often assumed that pitch-angle scattering is isotropic (see, e.g., Kirk \& Schneider 1988; Schneider \& Kirk 1989; Kirk \& Schneider 1989), in disagreement with the quasilinear result. It is also the purpose of the present article to investigate the validity of the assumption of isotropic scattering.

In Sect. 8 we consider some applications of our analytical results, namely:

1. cosmic rays from the Sun;

2. interstellar transport and steep turbulence spectra;

3. the Hillas limit and high energetic particles. 
and a discussion of the results is provided in Sect. 9 .

\section{Standard quasilinear theory}

The Fokker-Planck coefficient $D_{\mu \mu}$ used in Eq. (1) can be computed by employing the so-called Taylor-Green-Kubo formulation (TGK formulation, e.g. Taylor 1922; Green 1952; Kubo 1957)

$D_{\mu \mu}=\int_{0}^{\infty} \mathrm{d} t\langle\dot{\mu}(t) \dot{\mu}(0)\rangle$.

The acceleration parameter $\dot{\mu}(t)$ can be obtained from the Newton-Lorentz-equation whose parallel component reads

$\dot{\mu}=\frac{1}{R_{L}}\left(v_{x}(t) \frac{\delta B_{y}[\boldsymbol{x}(t), t]}{B_{0}}-v_{y}(t) \frac{\delta B_{x}[\boldsymbol{x}(t), t]}{B_{0}}\right)$

for purely magnetic fields ${ }^{1}$. Here we have used the unperturbed Larmor radius

$R_{L}=\frac{v}{\Omega}=\frac{v m c \gamma}{q B_{0}}=\frac{p c}{q B_{0}}$

with the unperturbed gyrofrequency $\Omega$, the particle mass $m$ and charge $q$, the mean magnetic field $B_{0}$, the speed of light $c$, and the particle momentum $p$.

The simplest method to compute the parameter $D_{\mu \mu}$ is the application of perturbation theory also known as quasilinear theory (Jokipii 1966). In this case the velocities $v_{x}(t)$ and $v_{y}(t)$ as well as the particle trajectories $\boldsymbol{x}(t)$ in Eq. (3) are replaced by the unperturbed particle orbit. A further assumption which is often used is that the stochastic magnetic fields are replaced by the so-called magnetostatic slab model for which we assume $\delta B_{i}(\boldsymbol{x})=\delta B_{i}(z)$. For the slab model the magnetic correlation tensor is given by

$P_{i j}(\boldsymbol{k})=\left\langle\delta B_{i}(\boldsymbol{k}) \delta B_{j}^{*}(\boldsymbol{k})\right\rangle=g^{\mathrm{slab}}\left(k_{\|}\right) \frac{\delta\left(k_{\perp}\right)}{k_{\perp}} \delta_{i j}$

with the (symmetric) turbulence spectrum $g^{\text {slab }}\left(k_{\|}\right)$. The combination of quasilinear theory and the magnetostatic slab model is also known as standard quasilinear theory. In this case we find (see, e.g., Teufel \& Schlickeiser 2002, 2003 for a detailed derivation of this formula)

$\begin{aligned} D_{\mu \mu}= & \frac{2 \pi v^{2}\left(1-\mu^{2}\right)}{B_{0}^{2} R_{L}^{2}} \int_{0}^{\infty} \mathrm{d} k_{\|} g^{\text {slab }}\left(k_{\|}\right) \\ & \times\left[R_{+}\left(k_{\|}\right)+R_{-}\left(k_{\|}\right)\right]\end{aligned}$

with the quasilinear resonance function

$R_{ \pm}\left(k_{\|}\right)=\pi \delta\left(v \mu k_{\|} \pm \Omega\right)$.

All parameters used in the present article are explained in Table 1 . The particle experiences only interaction with a certain wavenumber $v \mu k_{\|}=\Omega$ corresponding to $\mu R_{L} k_{\|}=1$. This scattering condition is known as gyroresonance. We find

$D_{\mu \mu}=\frac{2 \pi^{2} v\left(1-\mu^{2}\right)}{|\mu| B_{0}^{2} R_{L}^{2}} g^{\text {slab }}\left[k_{\|}=\left(|\mu| R_{L}\right)^{-1}\right]$.

In combination with Eq. (1) this formula can be used to compute the quasilinear parallel mean free path.

\footnotetext{
1 In the current article we neglect electric fields since they are less important for spatial diffusion. If one is interested in stochastic acceleration, however, electric fields are relevant (see, e.g., Schlickeiser 2002).
}

Table 1. Parameters used in the present article.

\begin{tabular}{ll}
\hline \hline Parameter & Symbol \\
\hline Inertial range spectral index & $\mathrm{s}$ \\
Energy range spectral index & $\mathrm{q}$ \\
Alfvén speed & $v_{A}$ \\
Slab bendover scale & $l_{\text {slab }}$ \\
Particle velocity & $v$ \\
Pitch-angle cosine & $\mu$ \\
Larmor radius & $R_{\mathrm{L}}$ \\
Gyrofrequency & $\Omega$ \\
Parallel wavenumber & $k_{\|}$ \\
Mean field & $B_{0}$ \\
Turbulence strength & $\delta B / B_{0}$ \\
Dimensionless rigidity & $R=R_{\mathrm{L}} / l_{\text {slab }}$ \\
\hline
\end{tabular}

\section{Second order quasilinear theory}

\subsection{The second order resonance function}

In this section we employ the second order theory of Shalchi (2005) in combination with the magnetostatic slab model. In the second order theory we no longer assume unperturbed orbits. Instead, quasilinear theory is employed in order to compute improved orbits. The improved orbits are then combined with Eqs. (1)-(3). Mathematically, the second order approach leads to a modified (broadend) resonance function (for a detailed derivation see Shalchi 2005) ${ }^{2}$

$R_{ \pm}^{S O Q L T}\left(k_{\|}\right)=\frac{\sqrt{\pi}}{v k_{\|}} \frac{B_{0}}{\delta B} \exp \left[-\frac{\left(v \mu k_{\|} \pm \Omega\right)^{2} B_{0}^{2}}{\left(v k_{\|} \delta B\right)^{2}}\right]$.

Here the resonance function has the form of a Gaussian function with the width

$\sigma^{2}=\left(\frac{v k_{\|} \delta B}{B_{0}}\right)^{2}$.

The quasilinear resonance function (see Eq. (7)) can be recovered by considering the limit $\sigma^{2} \rightarrow 0$. The physics of a nonlinear particle motion is illustrated in Fig. 1.

\subsection{An approximation for the resonance function}

The second order resonance function of Eq. (9) has the form

$R_{ \pm}^{S O Q L T}\left(k_{\|}\right)=\frac{\sqrt{\pi}}{\sigma} \exp \left[-\left(\beta_{ \pm}^{2} / \sigma^{2}\right)\right]$.

Please note that $\beta_{ \pm}=\beta_{ \pm}\left(k_{\|}\right)$. In Eq. (11) we have used the resonance condition

$\beta_{ \pm}=v \mu k_{\|} \pm \Omega$

and the width $\sigma$ as defined in Eq. (10). In principle the exponential (or Gaussian) function cuts off the wavenumber integral in Eq. (6). Furthermore, we have for the area under the resonance function

$\int_{-\infty}^{\infty} \mathrm{d} \beta_{ \pm} R_{ \pm}^{S O Q L T}\left(\beta_{ \pm}\right)=\pi$

2 The resonance function of Eq. (9) was obtained by Shalchi (2005) by combining a second order approach in combination with two mathematical approximations, namely a Late-Time-Approximation (LTA) and a $90^{\circ}$-approximation. These approximations were employed to ensure mathematical tractability. For a detailed explanation of these approximations and their justifications we refer to Shalchi $(2005,2009 b)$. 


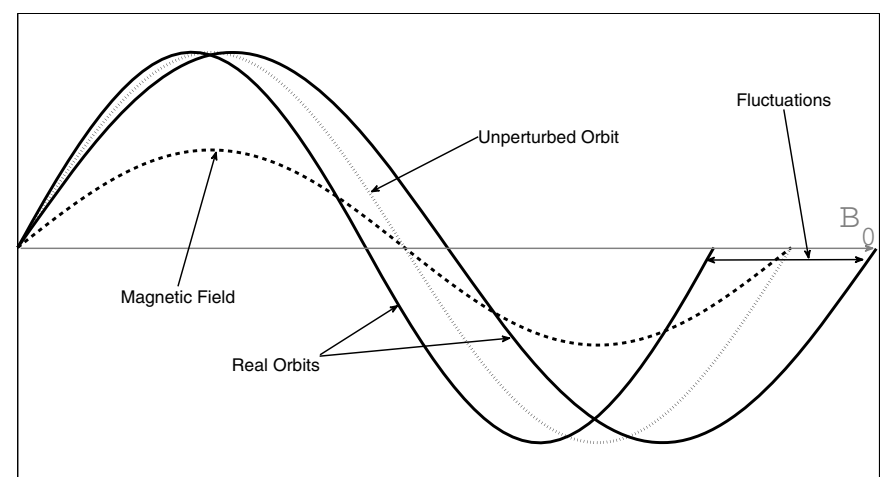

Fig. 1. The particle motion through the turbulent plasma. The turbulent magnetic field is represented by the dashed line. If there would be no interaction between the plasma and the cosmic rays, the particles would follow unperturbed orbits (dotted line). The latter trajectories are used in quasilinear theory. In reality, however, the particles experience scattering and, therefore, the true orbits decorrelate from the unperturbed motion (solid lines).

To achieve a simplification we approximate the resonance function by

$R_{ \pm}^{\text {SOQLT }}\left(k_{\|}\right)=\frac{\pi}{2 \sigma}\left\{\begin{array}{l}0 \text { for }\left|\beta_{ \pm}\right| \geq \sigma \\ 1 \text { for }\left|\beta_{ \pm}\right|<\sigma\end{array}\right.$

or in terms of the Heaviside stepfuntion $H$

$R_{ \pm}^{S O Q L T}\left(k_{\|}\right)=\frac{\pi}{2 \sigma} H\left(\sigma-\left|\beta_{ \pm}\right|\right) \cdot$

Equations (14) and (15) have similar properties in comparison with the original function (11). Furthermore, the function is similar to the heuristic ansatz of Völk (1975). In Fig. 2 the resonance functions of quasilinear theory, second-order theory (see Eq. (11)) and the approximation used in the present article (see Eqs. (14) and (15)) are visualized.

\subsection{The pitch-angle Fokker-Planck coefficient for the general case}

The pitch-angle Fokker-Planck coefficient from Eq. (6) has the form

$D_{\mu \mu}=\frac{2 \pi v^{2}\left(1-\mu^{2}\right)}{B_{0}^{2} R_{L}^{2}} I(\mu)$

with

$I(\mu)=I_{-}(\mu)+I_{+}(\mu)$

and

$I_{ \pm}=\int_{0}^{\infty} \mathrm{d} k_{\|} g^{\mathrm{slab}}\left(k_{\|}\right) R_{ \pm}\left(k_{\|}\right)$

To solve the integral with the approximation of Eq. (14) we have to split the integral. It is convenient to introduce the parameters

$$
\begin{aligned}
k_{ \pm} & =\frac{1}{R_{L}}\left(|\mu| \pm \delta B / B_{0}\right)^{-1} \\
k_{0} & =\frac{1}{|\mu| R_{L}} .
\end{aligned}
$$

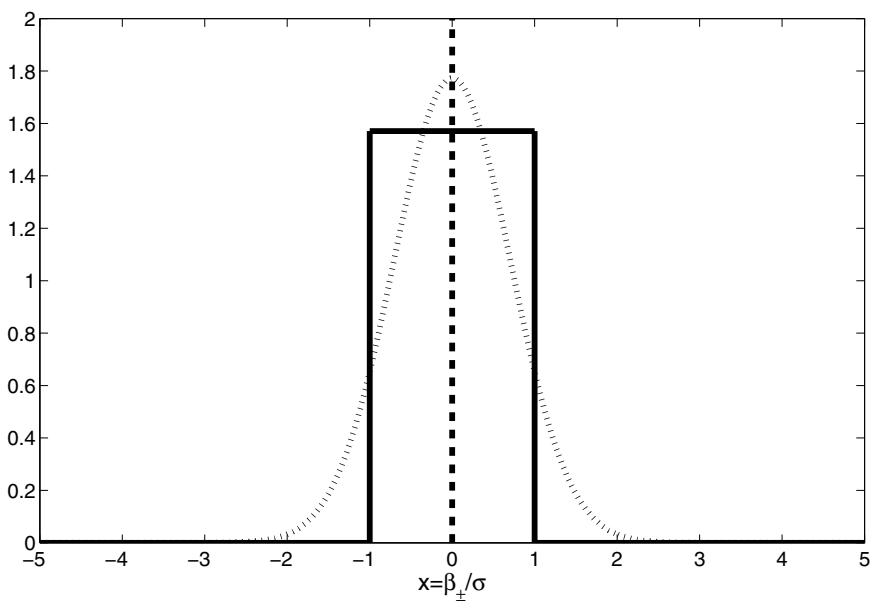

Fig. 2. The different resonance functions $\sigma R_{ \pm}$versus the resonance parameter $\beta_{ \pm} / \sigma$. The quasilinear resonance function (dashed line) is a $\delta$ function, whereas we find a Gaussian function within the second order theory (dotted line). In the present article we approximate the Gaussian function by a Heaviside stepfunction (solid line).

By combining Eqs. (15) and (18) we find after straightforward algebra

$$
\begin{aligned}
& I_{+}\left(|\mu|<\delta B / B_{0}\right)=\frac{\pi}{2} \int_{-k_{-}}^{\infty} \mathrm{d} k_{\|} g^{\text {slab }}\left(k_{\|}\right) \sigma^{-1} \\
& I_{+}\left(|\mu| \geq \delta B / B_{0}\right)=0
\end{aligned}
$$

The function $I_{-}$is more difficult to evaluate. We find

$$
\begin{aligned}
I_{-}(\mu)= & \frac{\pi}{2} \int_{0}^{k_{0}} \mathrm{~d} k_{\|} g\left(k_{\|}\right) \sigma^{-1} H\left(\sigma+v|\mu| k_{\|}-\Omega\right) \\
& +\frac{\pi}{2} \int_{k_{0}}^{\infty} \mathrm{d} k_{\|} g\left(k_{\|}\right) \sigma^{-1} H\left(\sigma-v|\mu| k_{\|}+\Omega\right) .
\end{aligned}
$$

To proceed we have to distinguish between the cases $|\mu| \geq \delta B / B_{0}$ and $|\mu| \leq \delta B / B_{0}$. We find after straighforward algebra

$I_{-}\left(|\mu|<\delta B / B_{0}\right)=\frac{\pi}{2} \int_{k_{+}}^{\infty} \mathrm{d} k_{\|} g^{\text {slab }}\left(k_{\|}\right) \sigma^{-1}$
$I_{-}\left(|\mu|>\delta B / B_{0}\right)=\frac{\pi}{2} \int_{k_{+}}^{k_{-}} \mathrm{d} k_{\|} g^{\text {slab }}\left(k_{\|}\right) \sigma^{-1}$.

By using the parameter

$\Gamma=\frac{\Omega}{v} \frac{B_{0}}{\delta B}=\frac{B_{0}}{R_{L} \delta B}$,

the total function $I=I_{-}+I_{+}$can be written as

$$
\begin{aligned}
I_{\text {nres }}\left(|\mu|<\delta B / B_{0}\right)= & \frac{\pi}{2 \Omega} \Gamma\left[\int_{-k_{-}}^{\infty} \frac{\mathrm{d} k_{\|}}{k_{\|}} g^{\text {slab }}\left(k_{\|}\right)\right. \\
& \left.+\int_{k_{+}}^{\infty} \frac{\mathrm{d} k_{\|}}{k_{\|}} g^{\text {slab }}\left(k_{\|}\right)\right] \\
I_{\text {qres }}\left(|\mu|>\delta B / B_{0}\right)= & \frac{\pi}{2 \Omega} \Gamma \int_{k_{+}}^{k_{-}} \frac{\mathrm{d} k_{\|}}{k_{\|}} g^{\text {slab }}\left(k_{\|}\right) .
\end{aligned}
$$

The case $|\mu|=\delta B / B_{0}$ can be obtained from both formulas by employing a limiting process. We find

$I\left(|\mu|=\delta B / B_{0}\right)=\frac{\pi}{2 \Omega} \Gamma \int_{k_{+}}^{\infty} \frac{\mathrm{d} k_{\|}}{k_{\|}} g^{\text {slab }}\left(k_{\|}\right)$. 
The first term in Eq. (24) is a non-resonant term and $I_{\text {qres }}$ can be called the quasi-resonant term. The reason will become clearer in the next section where we will discuss special cases.

\section{Special limits and cases}

Here we explore Eq. (24) for special limits and cases to recover previous results.

\subsection{The quasilinear limit}

In this paragraph we investigate the limit $|\mu| \gg \delta B / B_{0}$. In this case we have to use the quasi-resonant formula of Eq. (24). Therefore, we can approximate

$$
\begin{aligned}
I_{\text {qres }}\left(|\mu| \gg \delta B / B_{0}\right) & =\frac{\pi}{2 \Omega} \Gamma\left[g^{\text {slab }}\left(k_{\|}\right) k_{\|}^{-1}\right]_{k_{\|}=k_{0}} \int_{k_{+}}^{k_{-}} \mathrm{d} k_{\|} \\
& =\frac{\pi}{2 \Omega} \frac{\Gamma}{k_{0}} g^{\text {slab }}\left(k_{0}\right)\left[k_{-}-k_{+}\right] .
\end{aligned}
$$

With

$k_{-}-k_{+}=\frac{2}{R_{L}} \frac{\delta B / B_{0}}{\mu^{2}-\left(\delta B / B_{0}\right)^{2}}$

we find for $|\mu| \gg \delta B / B_{0}$

$I_{\text {qres }}\left(|\mu| \gg \delta B / B_{0}\right) \approx \frac{\pi}{v|\mu|} g^{\text {slab }}\left[k_{\|}=1 /\left(|\mu| R_{L}\right)\right]$

and the pitch-angle Fokker-Planck coefficient reads

$$
\begin{aligned}
D_{\mu \mu}\left(|\mu| \gg \delta B / B_{0}\right)= & \frac{2 \pi^{2} v\left(1-\mu^{2}\right)}{|\mu| B_{0}^{2} R_{L}^{2}} \\
& \times g^{\text {slab }}\left[k_{\|}=1 /\left(|\mu| R_{L}\right)\right]
\end{aligned}
$$

in agreement with Eq. (8). Obviously, quasilinear theory is valid so long as the restriction $|\mu| \gg \delta B / B_{0}$ holds. For pitch-angles satisfying $|\mu|>\delta B / B_{0}$, quasilinear theory could be approximately correct.

\subsection{Strong turbulence and $90^{\circ}$-scattering}

Now we investigate the limit $|\mu| \ll \delta B / B_{0}$. In this case we have

$k_{ \pm} \approx \pm \Gamma$

with the parameter $\Gamma$ defined in Eq. (23). Therefore the nonresonant term in Eq. (24) has the form

$I_{\text {nres }}\left(|\mu| \ll \delta B / B_{0}\right)=\frac{\pi}{\Omega} \Gamma \int_{\Gamma}^{\infty} \frac{\mathrm{d} k_{\|}}{k_{\|}} g^{\text {slab }}\left(k_{\|}\right)$

which is a pitch-angle independent result.

For strong turbulence $\left(\delta B \gg B_{0}\right)$ we always have $|\mu| \ll$ $\delta B / B_{0}$. In this case we find with Eq. (16) the form

$D_{\mu \mu}\left(\delta B \gg B_{0}\right)=\left(1-\mu^{2}\right) D$

with

$D=D_{\mu \mu}(\mu=0)=\frac{2 \pi^{2} \Omega}{B_{0}^{2}} \Gamma \int_{\Gamma}^{\infty} \frac{\mathrm{d} k_{\|}}{k_{\|}} g^{\text {slab }}\left(k_{\|}\right)$.

Equation (32) corresponds to an isotropic form (see later discussions). The parameter $D$ is the pitch-angle Fokker-Planck coefficient at $90^{\circ}$. The second order theory was already investigated analytically for $\mu=0$ in Shalchi (2005). In this paragraph we derived this limit from the general Eq. (24).

\section{Results for a realistic turbulence spectrum}

For the turbulence spectrum we employ the form introduced by Shalchi \& Weinhorst (2009)

$g^{\text {slab }}\left(k_{\|}\right)=\frac{D(s, q)}{2 \pi} \delta B^{2} l_{\text {slab }} \frac{\left|k_{\|} l_{\text {slab }}\right|^{q}}{\left[1+\left(k_{\|} l_{\text {slab }}\right)^{2}\right]^{(s+q) / 2}}$

with the normalization constant

$D(s, q)=\frac{\Gamma\left(\frac{s+q}{2}\right)}{2 \Gamma\left(\frac{s-1}{2}\right) \Gamma\left(\frac{q+1}{2}\right)}$.

In Eq. (34) we have used the bendover scale of the turbulence ${ }^{3}$ $l_{\text {slab }}$, the energy range spectral index $q$, and the inertial range spectral index $s$. The spectrum is correctly normalized for $q>$ -1 and $s>1$. In the following two paragraphs we investigate the quasi-resonant as well as the non-resonant case analytically for the spectrum defined in Eq. (34).

\subsection{The quasi-resonant case}

For the spectrum of Eq. (34) the quasi-resonant function derived in Eq. (24) becomes

$$
\begin{aligned}
I_{\text {qres }}\left(|\mu|>\delta B / B_{0}\right) & =\frac{D(s, q)}{4 \Omega} \delta B^{2} l_{\text {slab }} \Gamma \\
& \times \int_{k_{+}}^{k_{-}} \frac{d k_{\|}}{k_{\|}} \frac{\left|k_{\|} l_{\text {slab }}\right|^{q}}{\left[1+\left(k_{\|} l_{\text {slab }}\right)^{2}\right]^{(s+q) / 2}} .
\end{aligned}
$$

To proceed we employ the integral transformation $x=k_{\|} l_{\text {slab }}$ and split the integral to derive

$$
\begin{aligned}
I_{\text {qres }}\left(|\mu|>\delta B / B_{0}\right)= & \frac{D(s, q)}{4 \Omega} \delta B^{2} l_{\text {slab }} \Gamma \\
& \times\left[\int_{x_{+}}^{\infty} \mathrm{d} x \frac{x^{q-1}}{\left[1+x^{2}\right]^{(s+q) / 2}}\right. \\
& \left.-\int_{x_{-}}^{\infty} \mathrm{d} x \frac{x^{q-1}}{\left[1+x^{2}\right]^{(s+q) / 2}}\right]
\end{aligned}
$$

with

$x_{ \pm}=\frac{l_{\text {slab }}}{R_{L}} \frac{1}{|\mu| \pm \delta B / B_{0}}$.

The two integrals can be expressed by the hypergeometric function ${ }_{2} F_{1}(a, b ; c ; z)$ (see, e.g., Gradshteyn \& Ryzhik 2000)

$$
\begin{aligned}
& \int_{a}^{\infty} \mathrm{d} x \frac{x^{q-1}}{\left[1+x^{2}\right]^{(s+q) / 2}}= \\
& \frac{a^{-s}}{s}{ }_{2} F_{1}\left(\frac{s}{2}, \frac{q+s}{2} ; \frac{2+s}{2} ; \frac{-1}{a^{2}}\right) .
\end{aligned}
$$

With this formula we find

$$
\begin{aligned}
& I_{\mathrm{qres}}\left(|\mu|>\delta B / B_{0}\right)=\frac{D(s, q)}{4 s \Omega} \delta B^{2} l_{\text {slab }} \Gamma\left(\frac{R_{L}}{l_{\text {slab }}}\right)^{s} \\
\times & {\left[\left(|\mu|+\frac{\delta B}{B_{0}}\right)^{s}{ }_{2} F_{1}\left(\frac{s}{2}, \frac{q+s}{2} ; \frac{2+s}{2} ;-\xi^{2}\right)\right.} \\
- & \left.\left(|\mu|-\frac{\delta B}{B_{0}}\right)^{s}{ }_{2} F_{1}\left(\frac{s}{2}, \frac{q+s}{2} ; \frac{2+s}{2} ;-\xi^{2}\right)\right]
\end{aligned}
$$

3 The bendover or turnover scale denotes the frequency break between the large scales (energy range) and the intermediate scales (inertial range) of the turbulence. For the spectrum defined in Eq. (34) the bendover scale is directly proportional to the turbulence correlation length. 


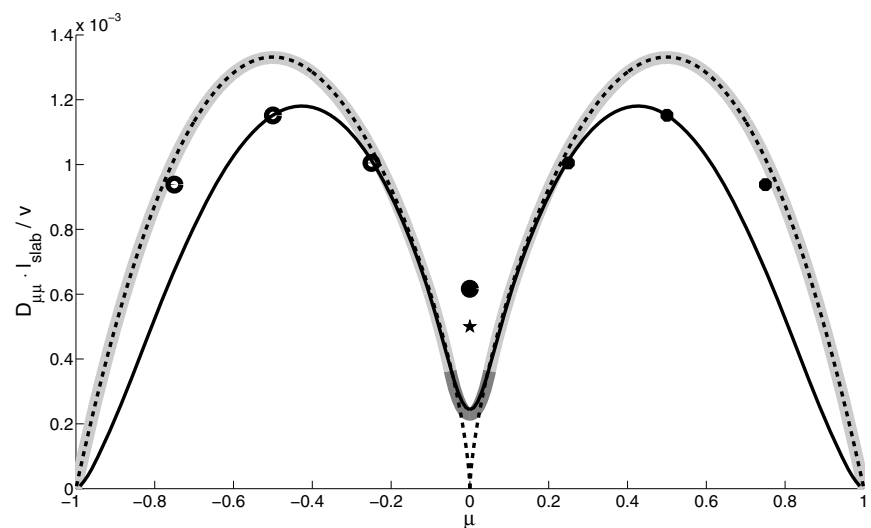

Fig. 3. Shown are the results of QLT (dotted line), SOQLT within the $90^{\circ}$-LTA (solid line), and simulations (Qin 2002, dots) for $D_{\mu \mu}$. Also shown is the more accurate SOQLT result without LTA (star) which is closer to the simulations. Furthermore, the analytical results derived in the present paper are shown. The resonant case is correct for $|\mu| \geq$ $\delta B / B_{0}$ (bright grey line) and the non-resonant case is valid for $|\mu| \leq$ $\delta B / B_{0}$ (dark grey line). In this plot, the rigidity is $R=0.0363$ and the strength of turbulence is assumed to be $\delta B / B_{0}=0.05$.

with

$\xi^{2}=\left(\frac{R_{L}}{l_{\text {slab }}}\right)^{2}\left(|\mu|+\frac{\delta B}{B_{0}}\right)^{2}$.

To proceed we consider $R_{L}|| \mu\left| \pm \delta B / B_{0}\right| \ll l_{\text {slab }}$. This case corresponds to the assumption of not too strong turbulence and low energetic particles. In this case the argument of the hypergeometric function is small $\left(\xi^{2} \ll 1\right)$ and we can use (see, e.g., Abramowitz \& Stegun 1974)

$$
{ }_{2} F_{1}(a, b ; c ;|z| \ll 1) \approx 1 .
$$

By combining Eqs. (37)-(42) and by using $R=R_{L} / l_{\text {slab }}$ we find

$$
\begin{aligned}
I_{\mathrm{qres}}\left(|\mu|>\delta B / B_{0}\right)= & \frac{D(s, q)}{4 s \Omega} \delta B^{2} l_{\mathrm{slab}} \Gamma R^{s} \\
& \times\left[\left(|\mu|+\frac{\delta B}{B_{0}}\right)^{s}-\left(|\mu|-\frac{\delta B}{B_{0}}\right)^{s}\right] .
\end{aligned}
$$

Note that this result is only valid for $|\mu|>\delta B / B_{0}$. From this result we can derive the quasilinear limit by considering $|\mu| \gg \delta B / B_{0}$

$I_{Q L T}(\mu)=\frac{D(s, q)}{2 v} l_{\text {slab }} \delta B^{2} R^{s}|\mu|^{s-1}$.

\subsection{The non-resonant case}

The calculations of the previous paragraph can be repeated for $|\mu|<\delta B / B_{0}$. In this case we have to use the non-resonant formula in Eq. (24) to derive

$$
\begin{aligned}
I_{\text {nres }}\left(|\mu|<\delta B / B_{0}\right) & =\frac{D(s, q)}{4 s \Omega} \delta B^{2} l_{\text {slab }} \Gamma R^{s} \\
& \times\left[\left(\frac{\delta B}{B_{0}}+|\mu|\right)^{s}+\left(\frac{\delta B}{B_{0}}-|\mu|\right)^{s}\right] .
\end{aligned}
$$

Except for the signs, Eq. (45) agrees with Eq. (43).

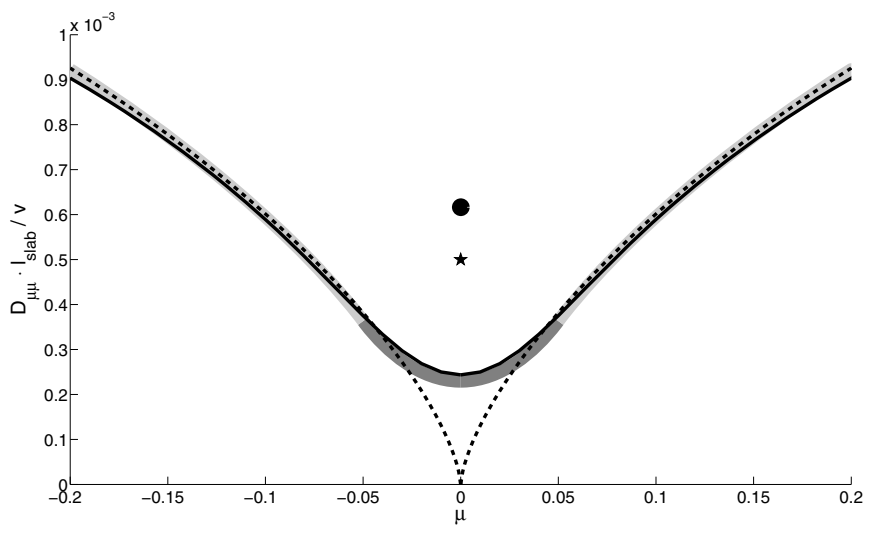

Fig. 4. Enlarge of Fig. 3 at small pitch-angle cosines.

\subsection{The general case}

Equations (43) and (45) can be combined to find for arbitrary $\mu$ the formula

$$
\begin{aligned}
I(\mu) & =\frac{D(s, q)}{4 s \Omega} \delta B^{2} l_{\text {slab }} \Gamma R^{s} \\
& \times\left[|| \mu\left|+\frac{\delta B}{B_{0}}\right|^{s}+\operatorname{sign}\left(\frac{\delta B}{B_{0}}-|\mu|\right)|| \mu\left|-\frac{\delta B}{B_{0}}\right|^{s}\right] .
\end{aligned}
$$

With Eq. (16) the pitch-angle Fokker-Planck coefficient becomes

$$
\begin{aligned}
D_{\mu \mu} & =\frac{\pi D(s, q)}{2 s}\left(1-\mu^{2}\right) \frac{v}{l_{\text {slab }}} R^{s-2} \frac{\delta B}{B_{0}} \\
& \times \sum_{n= \pm 1} \operatorname{sign}\left(\frac{\delta B}{B_{0}}+n|\mu|\right) \cdot|| \mu\left|+n \frac{\delta B}{B_{0}}\right|^{s}
\end{aligned}
$$

This formula can be applied for $R_{L}|| \mu\left| \pm \delta B / B_{0}\right| \ll l_{\text {slab. }}$ In the other case we have to consider the hypergeometric function of Eq. (40) in the limit of large arguments. In Figs. 3 and 4 we compare our analytical results with numerical results, QLT, and computer simulations.

\section{The parallel mean free path}

By using Eq. (1) we can compute the parallel mean free path. It is useful to consider again two different cases.

\subsection{The case $\delta B \gg B_{0}$}

Here we can use Eq. (1) to find approximately

$$
\begin{aligned}
\lambda_{\|} & =\frac{3 v}{4} \int_{0}^{1} \mathrm{~d} \mu \frac{\left(1-\mu^{2}\right)^{2}}{D_{\mu \mu}(\mu)} \\
& \approx \frac{3 v}{4} \int_{0}^{1} \mathrm{~d} \mu \frac{\left(1-\mu^{2}\right)^{2}}{D_{\mu \mu}\left(\mu \ll \delta B / B_{0}\right)} .
\end{aligned}
$$

By employing Eq. (47) for the pitch-angle Fokker-Planck coefficient in the limits $\mu \ll \delta B / B_{0}$, we derive

$\lambda_{\|}=\frac{s}{2 \pi D(s, q)} l_{\mathrm{slab}} R^{2-s}\left(\frac{B_{0}}{\delta B}\right)^{s+1}$.

This formula provides the behavior $\lambda_{\|} \sim\left(B_{0} / \delta B\right)^{s+1}$ in disagreement with the QLT result $\left(\lambda_{\|} \sim\left(B_{0} / \delta B\right)^{2}\right)$. 


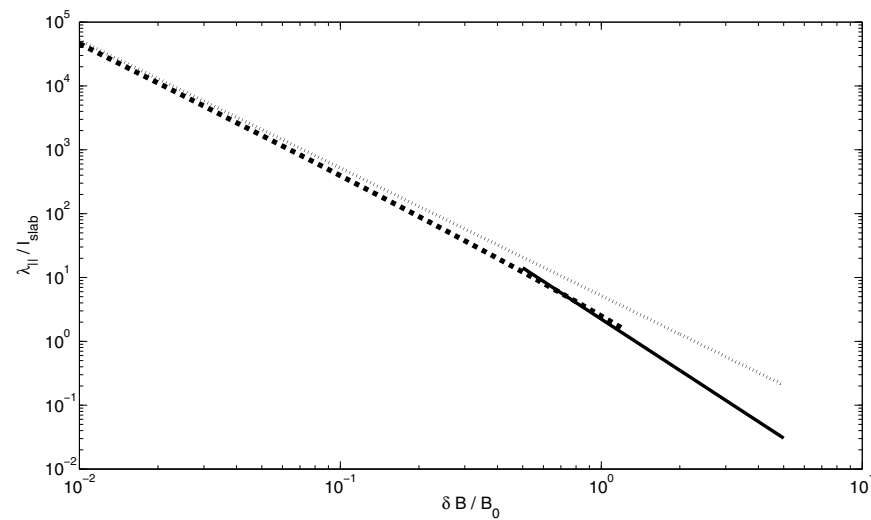

Fig. 5. The parallel mean free path computed by using QLT (dotted line) for $s=5 / 3$. Also shown are the analytical results of SOQLT, namely the weak turbulence solution (dashed line) of Eq. (51) and the strong turbulence solution (solid line) of Eq. (49).

\subsection{The case $\delta B \ll B_{0}$}

Here we can use Eq. (1) to find approximately

$$
\begin{aligned}
\lambda_{\|} & =\frac{3 v}{4} \int_{0}^{1} \mathrm{~d} \mu \frac{\left(1-\mu^{2}\right)^{2}}{D_{\mu \mu}(\mu)} \\
& =\frac{3 v}{4} \int_{0}^{\delta B / B_{0}} \mathrm{~d} \mu \frac{\left(1-\mu^{2}\right)^{2}}{D_{\mu \mu}\left(\mu \ll \delta B / B_{0}\right)} \\
& +\frac{3 v}{4} \int_{\delta B / B_{0}}^{1} \mathrm{~d} \mu \frac{\left(1-\mu^{2}\right)^{2}}{D_{\mu \mu}\left(\mu \gg \delta B / B_{0}\right)} .
\end{aligned}
$$

By employing Eq. (47) for the pitch-angle Fokker-Planck coefficient in the limits $\mu \ll \delta B / B_{0}$ and $\mu \gg \delta B / B_{0}$, we derive

$$
\begin{aligned}
\lambda_{\|}= & \frac{3}{2 \pi D(s, q)} l_{\text {slab }} R^{2-s}\left[\frac{1}{(2-s)(4-s)}\left(\frac{B_{0}}{\delta B}\right)^{2}\right. \\
& \left.+\frac{s(2-s)-1}{2(2-s)}\left(\frac{B_{0}}{\delta B}\right)^{s}\right] .
\end{aligned}
$$

The first term corresponds to the well-known quasilinear result. The second term is new and arises due to nonlinear effects. So long as the turbulent field is weak $\left(\delta B \ll B_{0}\right)$ and the inertial range spectral index satisfies $s<2$ the first term is dominant and quasilinear theory provides the correct result. For a weak turbulent field and the case $s>2$, corresponding to a steep inertial range spectrum, the second (nonlinear) term controls the parallel mean free path. In the latter case the parallel mean free path scales like $\lambda_{\|} \sim\left(B_{0} / \delta B\right)^{s}$. For a strong turbulent field we have to employ Eq. (49). In Fig. 5 we have shown the parallel mean free path versus the turbulence strength $\delta B / B_{0}$.

\section{Wave propagation effects}

\subsection{Parallel and anti-parallel propagating waves}

So far we have only discussed the magnetostatic case. In the current section we include plasma wave propagation effects by following the work of Schlickeiser (1989). We assume that there are only parallel and anti-parallel propagating shear Alfvén waves.

The magnetostatic pitch-angle Fokker-Planck coefficient has the form

$D_{\mu \mu}=\frac{2 \pi v^{2}\left(1-\mu^{2}\right)}{B_{0}^{2} R_{L}^{2}} I(|\mu|)$
Table 2. Plasmawave propagation versus nonlinearity.

\begin{tabular}{ll}
\hline \hline Case & Transport regime \\
\hline$\epsilon \ll|\mu| \& \delta B / B_{0} \ll|\mu|$ & Quasilinear magnetostatic regime \\
$\delta B / B_{0} \ll|\mu| \ll \epsilon$ & Quasilinear plasmawave regime \\
$\epsilon \ll|\mu| \ll \delta B / B_{0}$ & Nonlinear magnetostatic regime \\
$|\mu| \ll \epsilon \&|\mu| \ll \delta B / B_{0}$ & Nonlinear plasmawave regime \\
\hline
\end{tabular}

where we must distinguish the cases $|\mu| \geq \delta B / B_{0}$ and $|\mu| \leq$ $\delta B / B_{0}$. We include wave effects by employing the substitution

$|\mu| \rightarrow|\mu-j \epsilon|$

where we have used the (energy dependent) parameter $\epsilon=v_{A} / v$ with the Alfvén velocity $v_{A}$. The parameter $j$ is used to track the wave direction $(j=+1$ is used for forward and $j=-1$ for backward to the ambient magnetic field propagating Alfvén waves). If we assume equal intensities of parallel and anti-parallel propagating waves we can use

$I(|\mu|) \rightarrow I_{\text {wave }}(\mu)=I(|\mu-\epsilon|)+I(|\mu+\epsilon|)$.

We notice that

$I_{\text {wave }}(\mu=0)=2 I(\epsilon)$

and

$I_{\text {wave }}(\mu= \pm \epsilon)=I(2 \epsilon)$.

For $\mu=0$ as well as $\mu= \pm \epsilon$ we find a non-vanishing pitchangle Fokker-Planck coefficient. The question is whether wave propagation effects or nonlinear effects are more relevant. In the following paragraph we will investigate this issue.

\subsection{Wave versus nonlinear effects}

By combining Eqs. (46) and (54) we derive

$$
\begin{aligned}
I(\mu)= & \frac{D(s, q)}{4 s \Omega} \delta B^{2} l_{\text {slab }} \Gamma R^{s} \\
& \times \sum_{j, n= \pm 1} \operatorname{sign}\left(\frac{\delta B}{B_{0}}+n|\mu+j \epsilon|\right)|| \mu+j \epsilon\left|+n \frac{\delta B}{B_{0}}\right|^{s} .
\end{aligned}
$$

Mathematically, plasma wave propagation effects enter the function $I(\mu)$ in a similar way as the nonlinear effects. We can derive the parameter regimes for which wave effects are dominant and for which nonlinear effects have a stronger influence. It is convenient to define the characteristic velocity

$v_{c}=v_{A} \frac{B_{0}}{\delta B}$.

For low particles velocities $v \ll v_{c}$ we are in the wave dominated regime (since we have $\epsilon \gg \delta B / B_{0}$ ) and for higher velocities $v \gg$ $v_{c}$ we are in the nonlinear regime (since we have $\epsilon \ll \delta B / B_{0}$ ). Magnetostatic quasilinear theory is valid for $|\mu| \gg \epsilon$ and $|\mu| \gg$ $\delta B / B_{0}$ (see Table 2).

\section{Applications}

\subsection{Energetic particles from the sun}

To describe the motion of charged particles along the magnetic field of the Sun we can use the two-dimensional Fokker-Planck equation:

$\frac{\partial f}{\partial t}+v \mu \frac{\partial f}{\partial z}=\frac{\partial}{\partial \mu}\left(D_{\mu \mu} \frac{\partial f}{\partial \mu}\right)$ 
To proceed, we compute the spatial average

$F(\mu, t)=\int_{-\infty}^{+\infty} \mathrm{d} z f(\mu, z, t)$

and the Fokker-Planck equation becomes

$\frac{\partial F}{\partial t}=\frac{\partial}{\partial \mu}\left(D_{\mu \mu} \frac{\partial F}{\partial \mu}\right)$

By assuming that $\delta B \geq B_{0}$ and $v \gg v_{A}$ we can employ Eq. (32) and, therefore,

$\frac{\partial F}{\partial t}=D \frac{\partial}{\partial \mu}\left[\left(1-\mu^{2}\right) \frac{\partial F}{\partial \mu}\right]$.

This equation can be solved analytically without further assumptions. E.g., Shalchi (2006) has demonstrated that the solution of this equation can be expressed by Legendre polynomials $P_{l}(\mu)$ :

$F(\mu, t)=1+\sum_{l=1}^{\infty} \alpha_{l} P_{l}(\mu) \mathrm{e}^{-l(l+1) D t}$.

For sharp initial conditions $\left(\mu(t=0)=\mu_{0}\right)$ this becomes

$F(\mu, t)=1+\sum_{l=1}^{\infty}(2 l+1) P_{l}\left(\mu_{0}\right) P_{l}(\mu) \mathrm{e}^{-l(l+1) D t}$.

An interesting property is the anisotropy $A(t)$ which can be defined as

$A(t)=\frac{3 \int_{-1}^{+1} \mathrm{~d} \mu \mu F(\mu, t)}{\int_{-1}^{+1} \mathrm{~d} \mu F(\mu, t)}$.

With $P_{0}(\mu)=1, P_{1}(\mu)=\mu$, and

$\int_{-1}^{+1} \mathrm{~d} \mu P_{l}(\mu) P_{m}(\mu)=\frac{2}{2 m+1} \delta_{l m}$

we find

$A(t)=3 \mu_{0} \mathrm{e}^{-2 D t}=3 \mu_{0} \mathrm{e}^{-v t / \lambda_{\|}}$.

In the last step we have used Eq. (1) for the parallel mean free path. Analytical results such as Eq. (67) can be compared with measurements of solar particle events by spacecrafts such as Wind (see, e.g., Dröge \& Kartavykh 2009).

\subsection{Interstellar propagation and steep spectra}

In Lazar et al. (2003) and Spanier \& Schlickeiser (2005), the heating rate of the interstellar medium (ISM), especially the warm ionized medium, has been calculated. Within these two papers it was demonstrated that a steeper form of the turbulence spectrum $(s>2)$ could be reasonable. In this case we obtain by employing standard QLT an infinitely large parallel mean free path $\left(\lambda_{\|}=\infty\right)$. In Shalchi (2007) it has already been demonstrated the SOQLT is in agreement with simulations for such spectra. In the present section we compare our analytical finding with these previous results (see Fig. 6).

For the analytical results we can use Eq. (51) since we assume not too strong stochastic fields. These analytical forms are only valid for smaller rigidities. For high particle energies the analytical results deviate from the numerical finding. For weak turbulence and $s>2$ we can derive from Eq. (51) the formula

$\lambda_{\|}=\frac{3}{2 \pi D(s, q)} \frac{s(2-s)-1}{2(2-s)} l_{\text {slab }} R^{2-s}\left(\frac{B_{0}}{\delta B}\right)^{s}$.

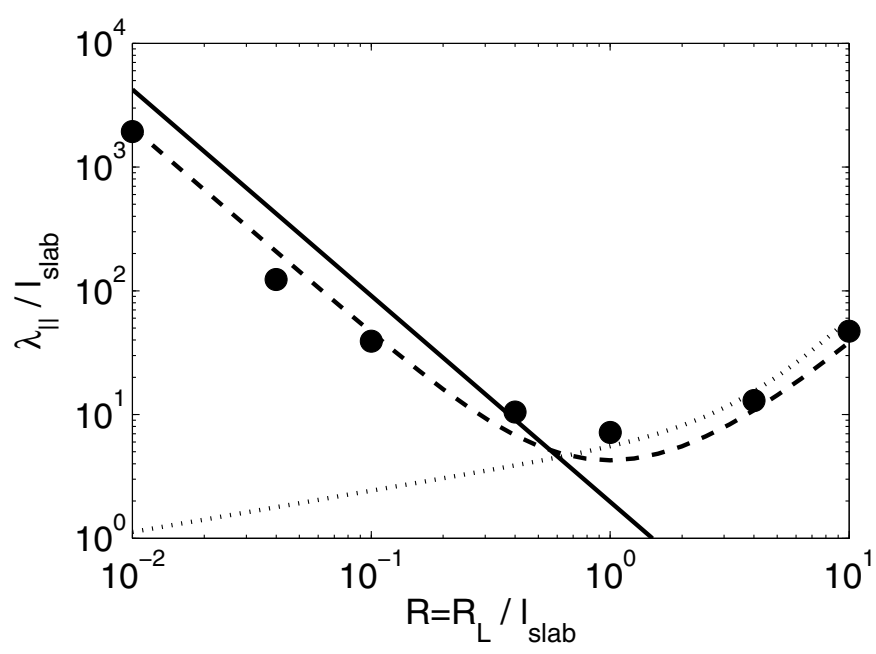

Fig. 6. $\lambda_{\|}$for pure slab geometry and steep spectra $(s=2+5 / 3)$. The simulations (dots) from Shalchi (2007) are compared with numerical SOQLT results (dashed line) and the analytical SOQLT result (solid line) obtained by employing Eq. (51). For sake of comparison we have also shown the QLT result for $s=5 / 3$ (dotted line). The magnetostatic QLT result for $s=2+5 / 3$ is $\lambda_{\|}=\infty$.

For strong turbulence we have to use Eq. (49). The pitch-angle Fokker-Planck coefficient for high particle rigidities can be derived from Eq. (40). In this case it is also straightforward to compute the parallel mean free path.

\subsection{The Hillas limit and high energetic cosmic rays}

In Shalchi et al. (2009a) we have investigated for the first time the propagation of ultrahigh energy particles within the framework of SOQLT. In this article we have computed numerically the pitch-angle Fokker-Planck coefficient and the parallel mean free path. As shown there, the Hillas limit ${ }^{4}$ is questionable.

It is the purpose of the present section to calculate analytically the pitch-angle Fokker-Planck coefficient of very high energetic particles. For simplicity we assume $\delta B \approx B_{0}$ and we can use Eq. (33). By using a spectrum with sharp cutoff at short wavenumbers we find

$D=\frac{2 \pi^{2} \Omega}{B_{0}^{2}} \Gamma \int_{\max \left[k_{\min }, \Gamma\right]}^{\infty} \frac{\mathrm{d} k_{\|}}{k_{\|}} g^{\text {slab }}\left(k_{\|}\right)$.

To evaluate this formula we assume $\Gamma<k_{\min }$ corresponding to

$\frac{R_{L}}{L_{\|}}>\frac{B_{0}}{\delta B} \approx 1$

with the largest scale of the turbulence $L_{\|}=k_{\min }^{-1}$. With the spectrum defined in Eq. (34) we can derive

$$
\begin{aligned}
D= & \pi \Omega D(s, q) l_{\text {slab }} \frac{\delta B^{2}}{B_{0}^{2}} \Gamma \\
& \times \int_{k_{\min }}^{\infty} \frac{\mathrm{d} k_{\|}}{k_{\|}} \frac{\left(k_{\|} l_{\text {slab }}\right)^{q}}{\left[1+\left(k_{\|} l_{\text {slab }}\right)^{2}\right]^{(s+q) / 2}} .
\end{aligned}
$$

${ }^{4}$ Within the framework of magnetostatic quasilinear theory, the resonance function is a sharp delta function. Since there exists a largest scale of turbulence $L_{\|}$, ultrahigh energy particles having a Larmor radius larger than this scale $\left(R_{L}>L_{\|}\right)$cannot be scattered and, therefore, they cannot be confined to the Galaxy. This limit is known as the Hillas limit (Hillas 1984). 
With the integral transformation $x=k_{\|} l_{\text {slab }}$ we derive

$D=\pi \Omega D(s, q) l_{\text {slab }} \frac{\delta B^{2}}{B_{0}^{2}} \Gamma Q\left(s, q, x_{\text {min }}\right)$

with $x_{\text {min }}=k_{\min } l_{\text {slab }}=l_{\text {slab }} / L_{\|} \ll 1$ and the integral

$Q\left(s, q, x_{\min }\right)=\int_{x_{\min }}^{\infty} \mathrm{d} x \frac{x^{q-1}}{\left[1+x^{2}\right]^{(s+q) / 2}}$.

By using Gradshteyn \& Ryzhik (2000) we can solve the integral to find

$Q\left(s, q, x_{\min }\right)=\frac{x_{\min }^{-s}}{s}{ }_{2} F_{1}\left(\frac{s}{2}, \frac{q+s}{2} ; \frac{2+s}{2} ;-x_{\min }^{-2}\right)$.

Finally, we find for the pitch-angle Fokker-Planck coefficient of ultrahigh energetic particles

$D_{\mu \mu}=\pi D(s, q) \frac{v l_{\text {slab }}}{R_{L}^{2}} \frac{\delta B}{B_{0}} Q\left(s, q, x_{\text {min }}\right)$.

By using Eq. (1) it is a simple matter to calculate analytically the parallel mean free path

$\lambda_{\|}=\frac{l_{\text {slab }}}{2 \pi D(s, q) Q\left(s, q, x_{\min }\right)} R^{2} \frac{B_{0}}{\delta B}$.

This result is valid for $R_{L} / L_{\|}>B_{0} / \delta B$ and strong stochastic fields. The quasilinear result for $R_{L} / L_{\|}>1$ is $\lambda_{\|}=\infty$.

Quasilinear theory predicts that the parallel mean free path of particles with $R_{L}>L_{\|}$is infinity. Consequently, the motion of high energy particle is scatter-free (ballistic) and, therefore, such particles cannot be confined to the Galaxy. According to Eq. (76), however, we find finite scattering within the framework of SOQLT. Therefore, we expect a finite confinement time of real particles in the Galaxy. For details we refer to Shalchi et al. (2009).

\section{Summary and conclusion}

In the present article we have revisited the problem of pitchangle scattering and parallel diffusion of charged particles. Previous investigations are based on the quasilinear approximation or on nonlinear theories. In the latter case only numerical results were available for the pitch-angle Fokker-Planck coefficient and the parallel mean free path. In Sect. 3 we have explored for the first time analytically the second order theory of Shalchi (2005a). By deriving general analytical expressions for the parameter $D_{\mu \mu}$, we have shown that the traditional quasilinear theory is correct for $|\mu| \gg \delta B / B_{0}$ and the assumption of isotropic scattering $D_{\mu \mu}=D\left(1-\mu^{2}\right)$ is valid for strong turbulence. This result confirms previous articles about diffusive shock acceleration (see, e.g., Kirk \& Schneider 1988; Schneider \& Kirk 1989; Kirk \& Schneider 1989). It should be noted, however, that for extremely strong turbulence one expects Bohm-diffusion (see Shalchi 2009a).

By employing the spectrum of Shalchi \& Weinhorst (2009a) we derived analytical forms for the Fokker-Planck coefficient $D_{\mu \mu}$ and the parallel mean free path $\lambda_{\|}$. The formulae for the latter parameter can also be used for strong turbulence and for steep $\operatorname{spectra}(s \geq 2)$. Quasilinear theory for parallel spatial diffusion is only valid for weak turbulence $\left(\delta B \ll B_{0}\right)$ and flat spectra $(s<2)$. For these spectra we have also introduced plasma wave propagation effects by following Schlickeiser (2002). For particle velocities satisfying $v \ll v_{c} \equiv v_{A} \delta B / B_{0}$ the quasilinear results obtained for the plasma wave model are correct since nonlinear effect are supressed. For $v \gg v_{c} \equiv v_{A} \delta B / B_{0}$ nonlinear effects are stronger and the magnetostatic model should provide a good approximation.

Analytical forms for the parameters $D_{\mu \mu}$ and $\lambda_{\|}$are very useful in the physics of cosmic rays. Therefore, we have presented some applications of our results (see Sect. 8). We have shown how energetic particles from the Sun can be described analytically be computing the anisotropy $A$. Such results can be compared with spacecraft observations such as Wind measurements (see, e.g., Dröge \& Kartavykh 2009). As a second example we computed the parallel mean free path of particles in the ISM by assuming steep turbulence spectra as suggested by Lazar et al. (2003) and Spanier \& Schlickeiser (2005). These analytical results complement the numerical work of Shalchi (2007). Standard quasilinear theory results in $\lambda_{\|}=\infty$ for such spectra. We have also considered the problem of ultrahigh energy cosmic rays. These results complement the numerical work of Shalchi et al. (2009a). We have derived for the first time a formula for the parallel mean free path of ultrahigh energy cosmic rays within SOQLT. According to this formula we have $\lambda_{\|} \sim R^{2} B_{0} / \delta B$ if the particle Larmor radius exceeds the largest scale of turbulence $\left(R_{L}>L_{\|}\right)$. We expect that our analytical results will lead to further interesting and important applications in astrophysics such as diffusive shock acceleration.

Acknowledgements. This research was supported by the Deutsche Forschungsgemeinschaft (DFG) under the Emmy-Noether Programm (grant SH 93/3-1) and project Schl 201/19-1. As a member of the Junges Kolleg, A. Shalchi also acknowledges support by the Nordrhein-Westfälische Akademie der Wissenschaften.

\section{References}

Abramowitz, M., \& Stegun, I. A. 1974, Handbook of Mathematical Functions (New York: Dover Publications)

Alania, M. V., \& Wawrzynczak, A. 2008, Astrophys. Space Sci. Trans., 4, 59 Dröge, W. 2000, Space Sci. Rev., 93, 121

Dröge, W., \& Kartavykh, Y. Y. 2009, ApJ, 693, 69

Earl, J. A. 1974, ApJ, 193, 231

Fletcher, L. 1997, A\&A, 326, 1259

Giacalone, J., \& Jokipii, J. R. 1999, ApJ, 520, 204

Gkioulidou, M., Zimbardo, G., Pommois, P., Veltri, P., \& Vlahos, L. 2007, A\&A, 462,1113

Goldstein, M. L. 1976, ApJ, 204, 900

Gradshteyn, I. S., \& Ryzhik, I. M. 2000, Table of integrals, series, and products (New York: Academic Press)

Green, M. S. 1951, J. Chem. Phys., 19, 1036

Hillas, A. M. 1984, ARA\&A, 22, 425

Jokipii, J. R. 1966, ApJ, 146, 480

Jones, F. C., Kaiser, T. B., \& Birmingham, T. J. 1973, PRL, 31, 485

Jones, F. C., Birmingham, T. J., \& Kaiser, T. B. 1978, Phys. Fluids, 21, 347

Kubo, R. 1957, J. Phys. Soc. Jpn., 12, 570

Kirk, J. G., \& Schneider, P. 1988, A\&A, 201, 177

Kirk, J. G., \& Schneider, P. 1989, A\&A, 225, 559

Lazar, M., Spanier, F., \& Schlickeiser, R. 2003, A\&A, 410, 415

Li, G., Zank, G. P., \& Rice, W. K. M. 2003, J. Geophys. Res., 108, 1082

Li, G., Zank, G. P., \& Rice, W. K. M. 2005, J. Geophys. Res., 110, A06104

Owens, A. J. 1974, ApJ, 191, 235

Schlickeiser, R. 1989, ApJ, 336, 243

Schlickeiser, R. 2002, Cosmic Ray Astrophysics (Berlin, Heidelberg: SpringerVerlag)

Schneider, P., \& Kirk, J. G. 1989, A\&A, 217, 344

Shalchi, A. 2005, Phys. Plasmas, 12, 052324

Shalchi, A. 2006, A\&A, 448, 809

Shalchi, A. 2007, J. Phys. G., 34, 209

Shalchi, A. 2009a, Astropart. Phys., 31, 237

Shalchi, A. 2009b, Nonlinear Cosmic Ray Diffusion Theories, Astrophysics and Space Science Library, 362 (Berlin: Springer)

Shalchi, A., \& Schlickeiser, R. 2005, ApJ, 626, L97 
Shalchi, A. \& Dosch, A. 2009, PRD, 79, 083001

Shalchi, A., \& Weinhorst, B. 2009, Adv. Space Res., 43, 1429

Shalchi, A., Bieber, J. W., Matthaeus, W. H., \& Schlickeiser, R. 2006, ApJ, 642, 2

Shalchi, A., Škoda, T., Tautz, R. C., \& Schlickeiser, R. 2009, PRD, 80, 023012 Spanier, F., \& Schlickeiser, R. 2005, A\&A, 436, 9

Tautz, R. C., Shalchi, A., \& Schlickeiser, R. 2008, ApJ, 685, L165

Taylor, G. I. 1922, Diffusion by continuous movement, Proceedings of the London Mathematical Society, 20, 196
Teufel, A., \& Schlickeiser, R. 2002, A\&A, 393, 703

Teufel, A., \& Schlickeiser, R. 2003, A\&A, 397, 15

Völk, H. J. 1973, Ap\&SS, 25, 471

Völk, H. J. 1975, Rev. Geophys. Space Phys., 13, 547

Yan, H., \& Lazarian, A. 2002, PRL, 89, 281102

Zank, G. P., Rice, W. K. M., \& Wu, C. C. 2000, J. Geophys. Res., 105, 25079

Zank, G. P., Li, G., Florinski, V., et al. 2006, J. Geophys. Res., 111, A06108 